\title{
Certified Nurse Practitioner
}

National Cancer Institute

\section{Source}

National Cancer Institute. Certified Nurse Practitioner. NCI Thesaurus. Code C71288.

A registered nurse who meets the requirements for licensure as an advanced practice nurse. A CNP can order diagnostic tests, prescribe medications and drugs, and administer medications and drugs. 